Vidyodaya J., of Sci., (1992) Vol. 4, No. 1, pp: 137 - 145

\title{
A NUMERICAL TECHNIQUE TO DETERMINE THE MAGNETIZATION OF A TWO DIMENSIONAL BODY
}

\author{
D. A. Tantrigoda \\ Department of Physics. \\ University of Sri Jayewardenepura, \\ Nugegoda, Sri Lanka.
}

Received on: 17-06-1992

Accepted on : $09-08-1992$

\begin{abstract}
A simple numerical technique that permits the determination of the magnetization of a two dimentional body, producing a magnetic anomaly as well as a gravity anomaly, is presented. This technique involves the comparison of the Fourier transform of the magnetic anomaly, computed starting from the gravity anomaly using the Poisson relationship, with the Fourier transforn of the observed magnetic anomaly and can easily be extended to the three dimensional case (to determine the direction of magnetization only) As most of the magnetized basic rocks that produce magnetic anomalies also produce gravity anomalies, the present technique has useful practical applications in the interpretation of magnetic anomalies.
\end{abstract}

Key words: Numerical Technique, Magnetic Anomaly, Poisson Relationship.

\section{Introduction}

Numerical techniques are available to determine the shape and size of a subsurface structure, causing a magnetic anomaly, using the measurements of the anomaly made on the surface on the earth or above (Bott, 1973). This is generally known as the solving of the inverse problem in magnetics. The magnetization of the causative body is an important parameter in this calculation. The magnitude of magnetization of a rock sample can be obtained relatively easily determining its susceptibility. However, the measurement of the direction of magnetization, when the remanent component is not negligible, requires the use of instruments that are normally used in paleomagnetic work.

This paper presents a simple numerical technique to determine the magnetization of a two dimensional body which produces both gravity and magnetic anomalies. Most of the basic rocks that produce magnetic anomalies have positive density contrasts compared to the country rocks and therefore produce positive gravity anomalies. Examples for such basic rocks can be found in the Teitiary volcanic centres such as Mull and Skye in the NW Scotland (Bott and Tantrigoda, 1986; Tantrigoda, 1988) and in the Blackstones Bank in the sea areas of Hebridees (Tantrigoda, 1986). The present technique, therefore has useful practical applications. This technique requires the 
density contrast of the causative body and does not make any assumptions regarding its shape. Further, this can easily be extended to the three dimensional case, but only to determine the direction of magnetization provided the density contrast as well as the magnitude of the magnetization of the causitive body are available.

\section{Theory}

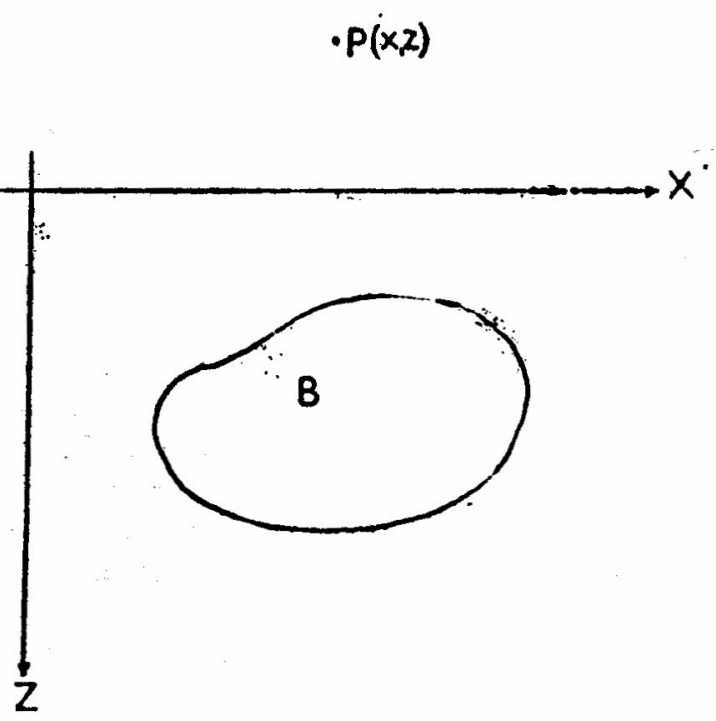

Fig. 1 Coordinate system used in the derivation of the technique and the location of the point $\mathrm{P}$ and the body $\mathrm{B}$.

Let $\mathrm{XZ}$ be a two dimensional Cartesean coordinate system with the $Z$-axis pointing vertically downwards and $V(x, z)$ and $U(x, z)$ be the magnetic and gravity potentials produced by a body $B$ situated below the $X$-axis at a point $P(x, z)$ above the $X$-axis (fig. 1). The magnetic potential $V(x, z)$ and the gsavity potential $\mathrm{U}(\mathrm{x}, \mathrm{z})$ are connected by the Poisson relationship.

$$
V=-\frac{J_{\cdot} \nabla U}{4 \pi \rho G}
$$

Where $\mathbf{J}$ and $\rho$ are the magnetization vector and the density contrast and $\mathbf{G}$ is the gravitational constant. If $\mathbf{s}$ is the direction of earth's total field, then the magnetic anomaly $f(x, z)$ produced at $P$ can be writted as,

$$
f(x, z)=\frac{\mu_{0}}{4 \pi \rho G}\left\{\left(-s_{x}{ }^{J} x^{+s} z_{z}\right) \frac{\partial^{2} U}{\partial z^{2}}\left(s_{x} J+s_{z} J_{x}\right) \frac{\partial^{2} U}{\partial x^{-} \delta}\right\}
$$


If $g(x, z)$ is the gravity amomaly produced by $B$ at the same point $P$, then (2) can be written as,

$$
f(x, z)=\frac{\mu_{0}}{4 \pi \rho G}\left\{\left(-s_{x}{ }^{\top} x^{+s} z^{J} z^{\prime}\right) \frac{\partial g}{\partial z}+\left(s_{x} J^{J}+s_{z}{ }^{J}\right) \frac{\partial g}{\partial x}\right\}
$$

Let us assume that we are intending to analyse a magnetic anomaly $f(x, f)$ and a gravity anomaly $g(x, f)$ measured over a lenght $L$ and sampled at $\mathrm{N}$ equal intervals. Since the giavity amomaly and magnetic anomaly both satisfy the Laplace equation in the Cartesean coordinates, $f(x, z)$ and $g(x, z f)$ can be written as (Bott, 1973).

$$
\begin{aligned}
& f(x, z)=\sum_{k=0}^{N-1} F(k) \exp \left[\frac{2 \pi i k x}{L}\right] \quad \exp \left[\frac{2 \pi k z}{L}\right] \\
& g(x, z)=\sum_{k=0}^{N-1} G(k) \exp \left[\frac{2 \pi i k x}{L}\right] \quad \exp \left[\frac{2 \pi k z}{L}\right]
\end{aligned}
$$

Where, $F(k)$ and $G(k)$ are complex Fourier coefficients of the magnetic and gravity anomalies, Let $X_{m}(k)$ and $Y_{m}(k)$ be the real and imaginary parts of the Fourier transform of the magnetic anomaly and $X_{g}(k)$ and $Y_{g}(k)$ be the real and imaginary parts of the Fourier transform of the gravity anomaly. Substituting for $f(x, z)$ and $g(x, z)$ in (3) from (4) and (5) and expressing $F(k)$ and $G(k)$ in terms of their real and imaginary parts we get, 


$$
\begin{aligned}
& \sum_{k=0}^{N-1}\left\{x_{m}(k)+i Y_{m}\left(\operatorname{lexp}\left\{\frac{2 \pi i k}{L}\right\}-1\left[\frac{2 \pi i z}{L}\right]^{n}\right.\right. \\
& =\sum_{K=0}^{N-1}\left(\frac{\mu_{0} k}{2 \rho G L}\right)\left(\left\{-J_{x}\left(X_{g}(k) s_{x}+Y g(k) s_{z}\right)+J_{z}\left(X_{g}(k) s_{z}-Y_{g}(k) s_{x}\right)\right\}\right. \\
& \left.+i\left\{J_{x}\left(x_{g}(k) s_{z}-Y_{g}(k) s_{x}\right)+J_{z}\left(x_{g}(k) s_{x}+Y_{g}(k) s_{z}^{\prime \prime}\right)\right\}\right] \\
& \exp \left\{\frac{2 \pi i k x}{L}\right\} \exp \left\{\frac{2 \pi k z}{L}\right\}
\end{aligned}
$$

The right hand side of the above equation gives the real and imaginary parts of the Fourier coefficients of various frequencies $k$ of the magnetic anomaly calculated starting trom the gravity anomaly, at a set of $\mathrm{N}$ points, using the Poisson relationship. The left hand side of the equation gives real and imaginary parts of the Fourier coefficients of the magnetic anomaly for different frequencies $k$ computed directly form the observed magnetic anomaly at the same set of $\mathrm{N}$ points as in the previous case. Both sides of (6), therefore gives the real and imaginary components of the Fourier transform of the same magnetic anomaly. By equating the real and imaginary parts of the Fourier coefficients on the left hand side to those of the right hand side for different $k$ we get,

$$
\begin{gathered}
-J_{x}\left\{x_{g}(k) s_{x}+Y_{g}(k) s_{z}\right\}+J_{z}\left\{x_{g}(k) s_{z}-Y_{g}(k) s_{x}\right\}=\left(\frac{2 \rho G l}{\mu_{0} k}\right) X_{m}(k) \\
J_{x}\left\{x_{g}(k) s_{z}-Y_{g}(k) s_{x}\right\}+J_{z}\left\{X_{g}(k) s_{x}+Y_{g}(k) s_{z}\right\}=\left(\frac{2 \rho G L}{\mu_{0} k}\right) Y_{m}(k) \\
\text { for } k=1,2,3 \ldots \ldots \ldots \ldots \ldots \ldots . . . .2
\end{gathered}
$$


There is no point in equating terms corresponding to $k>N / 2$ as the real and imaginary parts of a Fourier transform of a real function are symmetric and antisymmetric respectively. No new information can be obtainhd by equating the terms having $k$ greater than the folding frequency. Equations (7) and (8) can be solved immediately to obtain $\mathrm{J}_{x}$ and $\mathrm{J}_{z}$ corresponding to different $k$ values. As it is explained earlier the present technique gives $N / 2$ sets of values for $\mathrm{J}_{\mathrm{x}}$ and $\mathrm{J} £$ and this will be discussed in more detail later.

\section{Testing of The Method}

A computer routine which uses a fast Fourier transform algorithem (Cooley ane Tukey, 1965) has been written to calculate the horizontal $\left(\mathrm{J}_{\mathrm{x}}\right)$ and vertical $\left(\mathrm{J}_{\mathrm{z}}\right)$ components of the magnetization vector and has been used to test the validity of the preceeding method. Magnetic and gravity anomalies due to a two dimensional rectangular body having known $J_{x}$ and $J_{z}$ values and a density contrast were calculated using Bott (1973) (Fig. 2). These gravity and magnetic anomaly values were used as input data to the above programme and two sets of values were calculated for $J_{x}$ and $J_{z}$ corresponding to different $\mathrm{k}$ values (ie. for different frequencies). As can be seen from the Table 1 that calculated $J_{x}$ and $J_{z}$ values are correct within the error range of 10 percent corresponding to $k=2,3 \ldots \ldots 7$. However the error becomes significantly large for larger $k$ values.

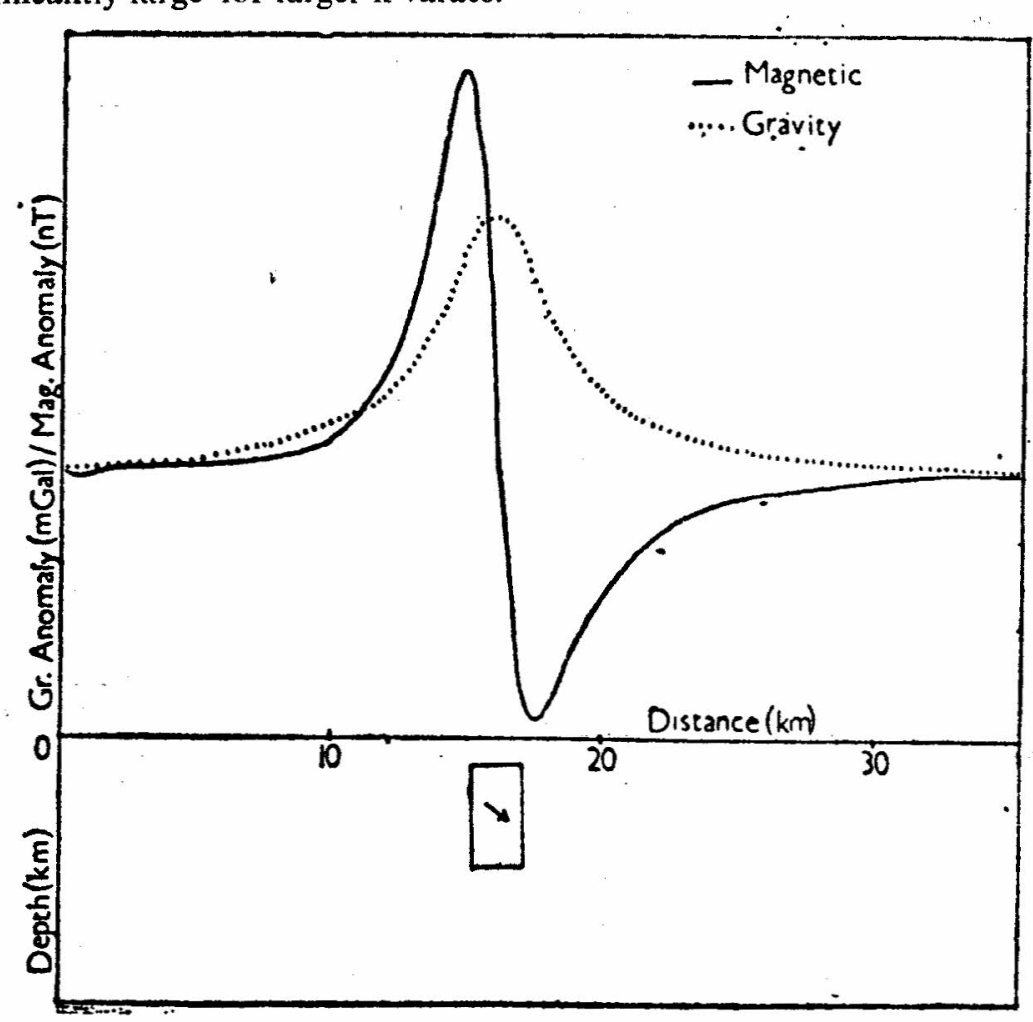

Fig. 2 Gravity and magnetic anomaly values (due to a 2D rectangular body) used for the testing of the technique. 
Table 1 Calculated values of $\mathrm{J}_{\mathrm{x}}$ and $\mathrm{J}_{\mathrm{z}}$ for different $\mathrm{k}$ values.

\begin{tabular}{|c|c|c|c|c|}
\hline$k$ & $J_{z}\left(A m^{-1}\right)$ & $\begin{array}{l}\text { Percentage Error } \\
\left(\mathrm{Am}^{-1}\right)\end{array}$ & $J_{\mathbf{z}}\left(A m^{-1}\right)$ & $\begin{array}{l}\text { Percentage Error } \\
\quad\left(\mathrm{Am}^{-1}\right)\end{array}$ \\
\hline 1 & 2.20 & 1.4 & 2.41 & 3.4 \\
\hline 2 & 2.16 & 0.3 & 2.55 & 2.1 \\
\hline 3 & 2.16 & 0.3 & 2.45 & 2.2 \\
\hline 4 & 2.18 & 0.6 & 2.57 & 2.9 \\
\hline 5 & 2.14 & 1.0 & 2.40 & 3.9 \\
\hline 6 & 2.20 & 1.4 & 2.65 & 6.1 \\
\hline 7 & 2.11 & 2.4 & 2.29 & 8.0 \\
\hline 8 & 2.23 & 3.1 & 2.85 & 14.1 \\
\hline 9 & 2.05 & 4.9 & 2.08 & 16.8 \\
\hline 10 & 2.22 & 2.3 & 3.43 & 37.2 \\
\hline 11 & 1.93 & 10.8 & 1.63 & 34.8 \\
\hline 12 & 0.92 & 57.6 & 5.45 & 117.9 \\
\hline 13 & 1.54 & 29.1 & 0.83 & 66.9 \\
\hline 14 & -2.71 & 225.1 & 0.55 & 77.9 \\
\hline 15 & 0.54 & 75.2 & -0.06 & 100.3 \\
\hline 16 & -0.06 & 103.1 & -0.15 & 106.1 \\
\hline 17 & -0.53 & 124.4 & -0.11 & 104.3 \\
\hline 18 & 0.46 & 78.5 & -0.03 & 101.6 \\
\hline 19 & -.88 & 140.6 & 0.12 & 95.2 \\
\hline 20 & 0.54 & 74.9 & -0.04 & 101.6 \\
\hline 21 & -0.80 & 137.3 & -0.23 & 190.9 \\
\hline 22 & 0.47 & 78.3 & -0.07 & 103.0 \\
\hline 23 & -0.62 & 128.8 & -0.24 & 90.3 \\
\hline 25 & 0.35 & 83.9 & -0.10 & 103.9 \\
\hline 26 & -0.44 & 120.6 & 0.23 & 90.9 \\
\hline 27 & 0.23 & 89.3 & -0.09 & 103.8 \\
\hline 28 & 0.30 & 113.9 & 0.21 & 91.5 \\
\hline 29 & 0.14 & 93.4 & -0.08 & 103.8 \\
\hline 30 & -0.19 & 108.7 & -0.20 & 91.8 \\
\hline 31 & 0.09 & 95.5 & -0.06 & 102.5 \\
\hline 32 & -0.07 & 103.4 & 0.22 & 91.2 \\
\hline
\end{tabular}


Normally magnetic measurements consists of high frequency instrumental noise and also noise due to the narrow near surface magnetic intrusive bodies. Therefore to see how successfully the present technique can cope up with such situations, the test was repeated after adding 5 percent random noise to the both gravity and magnetic anomalies due to the two dimensional body mentioned earlier. The results are depicted in Table 2 and as can be seen that the calculated $\mathrm{J}_{\mathrm{x}}$ and $\mathrm{J}_{\mathrm{z}}$ values are correct within 10 percent error for $\mathrm{k}=1,2,3,4$.

Table 2 Calculated values of $\mathrm{J}_{\mathrm{x}}$ and $\mathrm{J}_{\mathrm{z}}$ for different $\mathrm{k}$ values. $5 \%$ random noise has been added to both gravity and magnetic anomalies.

\begin{tabular}{|c|c|c|c|c|}
\hline$k$ & $\left(J x A m^{-1}\right)$ & $\begin{array}{c}\text { Percentage Error } \\
\left(A m^{-1}\right)\end{array}$ & $J z\left(A m^{-1}\right)$ & $\begin{array}{l}\text { Percentage Error } \\
\left(\mathrm{Am}^{-1}\right)\end{array}$ \\
\hline 1 & 2.21 & 1.9 & 2.43 & 2.6 \\
\hline 2 & 2.20 & 1.4 & 2.54 & 1.0 \\
\hline 3 & 2.21 & 2.1 & 2.39 & 4.4 \\
\hline 4 & 2.28 & 5.5 & 2.44 & 2.5 \\
\hline 5 & 2.29 & 6.2 & 2.23 & 10.5 \\
\hline 6 & 2.46 & 13.7 & 2.37 & 5.3 \\
\hline 7 & 2.41 & 11.1 & 1.86 & 25.4 \\
\hline 8 & 3.16 & 46.1 & 2.33 & 6.9 \\
\hline 9 & 2.65 & 22.3 & 1.58 & 36.9 \\
\hline 10 & 3.66 & 69.2 & 0.46 & 81.6 \\
\hline 11 & 1.66 & 23.3 & 0.10 & $104.1^{\circ}$ \\
\hline 12 & 1.59 & 26.5 & -0.20 & 108.1 \\
\hline 13 & 0.99 & 54.4 & -0.11 & 104.6 \\
\hline 14 & 0.72 & 66.6 & 0.27 & 88.9 \\
\hline 15 & 0.08 & 96.2 & -0.16 & 106.2 \\
\hline 16 & -0.38 & 117.4 & -0.27 & 110.8 \\
\hline 17 & -0.16 & 107.5 & 0.07 & 97.2 \\
\hline 18 & 0.21 & 90.3 & 0.19 & 92.3 \\
\hline 19 & 0.08 & 96.5 & 0.49 & 80.3 \\
\hline 20 & 0.61 & 71.9 & 0.04 & 101.5 \\
\hline 21 & 0.34 & 84.2 & 0.36 & 85.5 \\
\hline 22 & 0.22 & 89.6 & 0.02 & 99.2 \\
\hline 23 & -0.26 & 111.9 & 0.02 & 99.2 \\
\hline 25 & -0.10 & 104.8 & -0.15 & 106.1 \\
\hline 26 & -0.03 & 101.4 & 0.11 & 95.5 \\
\hline 27 & 0.22 & 89.7 & 0.16 & 93.7 \\
\hline 28 & 11.17 & 415.8 & 0.24 & 90.3 \\
\hline 29 & 0.03 & 98.5 & -0.44 & 117.7 \\
\hline 30 & -0.13 & 105.9 & -0.20 & 108.7 \\
\hline 31 & 0.06 & 102.6 & -0.03 & 101.3 \\
\hline 32 & -0.01 & 100.5 & 0.02 & 09.1 \\
\hline
\end{tabular}




\section{Discuscion}

A simple numerical technique which permits the determination of the magnetization of a two dimensional body producting a gravity anomaly as well as a magnetic anomaly is presented. The technique was first tested using gravity and magnetic anomalies produced by a two dimensional body and then further tested using anomalies due to the same body after adding 5 percent random noise to simulate high frequency noise and found working satisfactorily. This can easily be extended to the three dimensional case only to determine the direction of magnetization provided density to magnetization (magnitude) ratio is known.

If the input gravity and magnetic data are sampled at equal intervals at $N$ points, then this method gives $N / 2$ values for $J_{x}$ and a same number of values for $J_{z}$ corresponding to $k=1,2 \ldots \ldots \ldots N / 2$. As it was pointed out earlier, only the $J_{\mathbf{x}}$ and $\mathbf{J}_{\mathbf{z}}$ values corresponding to first few $k$ values are correct. This technique is based on the comparison of Fourier coefficients of the magnetic anomaly calculated starting from the gravity anomaly, with that of the measured magnetic anomaly for different frequencies (ie. for different $k$ values). The Fourier coefficients of higher frequencies normally get modified due to the truncation effect and aliasing (Brigham, 1974). Calcuations carried out comparing incorrect coeficients naturally give incorrect values for magnetization components. The error due to aliasing can be removed by increasing the number of sampling intervals and that due to truncation effect may be reduced to a reasonable level by passing both the gravity and magnetic anomalies through a suitable filter (eg. Hanning filter). However, it is not necessary to do this, as one can obtain satisfactory results by taking the average of $J_{x}$ and $J_{z}$ values which do not undergo more than 5 percent change compared to the $J_{x}$ and $J_{z}$ values for $k=1$.

The numerical technique presented in this paper will be useful in the interpretation of magnetic anomalies and may also provide information of paleomagnetic significance. 


\section{References}

Bott, M. H. P. and Tantrigoda, D. A., 1987. Iaterpretation of gravity and magnetic anomalies over the Mull Tertiary intrusive complex, NW Scotland, Journal of the Geological Society London, 144, 17-28.

Bott M.H.P., 1973. Inverse methods in the interpretation of magnetic and gravity anomalies, in "Methods of Computational Physics, 13" (edited by B. A. Bolt), 133-162.

Brigham E.0.1974. The Fast Fourier Transform, Prentice Hall, N.J., 255pp.

Cooly J.W. and Tukey J.W., 1965. An algorithm for mechine calculation of the complex Fourier series, Mathematical Computations 19, 297-301.

Tantrigoda, D.A., 1988. Interpretation of magnetic anomalies over the Skye Tertiary intrusive complex, Scott. J. Geol., 24(3), 215-221.

Tantrigoda, D.A., 1986. Interpretation of magnetic anomalies over the Blackstones Bank igneous centre, Scott. J. Geol., 22(1), 127-131. 